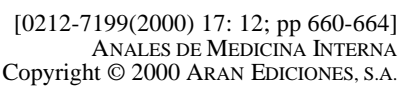

Copyright (C) 2000 ARAN EDICIONES, S.A.

AN. MED INTERNA (Madrid) Vol. 17, N. ${ }^{\circ} 12$, pp. 660-664, 2000

\title{
Tratamiento de las hepatitis virales crónicas B y C según la medicina basada en la evidencia
}

\author{
D. LEDRO CANO, M. GÓMEZ PARRA, M. JIMÉNEZ SÁENZ ,D. LEDRO \\ MOLINA*, J. M. HERRERÍAS GUTIÉRREZ \\ Servicios de Aparato Digestivo y Medicina Interna A*. Área Hospitalaria Virgen \\ Macarena. Sevilla
}

\author{
EVIDENCE-BASED MEDICINE TREATMENT OF CHRONIC VIRAL \\ HEPATITIS B AND C
}

\begin{abstract}
RESUMEN
En todo el mundo, la hepatitis viral es la principal causa de ictericia, enfermedad hepática crónica, cirrosis y hepatocarcinoma. Aunque se han realizado importantes avances en el tratamiento y en la prevención, no existe un tratamiento totalmente satisfactorio para cada una de estas dos enfermedades.

Ambas representan un porcentaje elevado de la etiología de las hepatitis virales y tienen una alta tendencia a la cronicidad y al desarrollo de cirrosis, conllevando gran consumo de recursos sanitarios. Por otra parte los tratamientos son prolongados y con fármacos de precio elevado. Por ello es necesario aplicar la medicina basada en la evidencia en este tipo particular de patología para alcanzar la mejor relación coste/beneficio. En la presente revisión, analizamos los diferentes fármacos y regímenes de tratamiento empleados en las hepatitis crónicas virales B y C, así como las respuestas obtenidas.
\end{abstract}

PALABRAS CLAVE: Ensayos controlados aleatorizados. Hepatitis crónica C. Hepatitis crónica B. Tratamiento.

\begin{abstract}
Worldwide, viral hepatitis is the most common cause of jaundice, chronic liver disease, cirrhosis and hepatocellular carcinoma. Although mayor advances have been made in the field of treatment and prevention, there is not a totally satisfactory treatment for each of both diseases. They account for a high percentage of the etiology of viral hepatitis and have a tendency towards chronicity and developing cirrhosis, resulting in a tremendous waste of medical resources. On the other hand, their tre atments are long-term ones and the drugs, which are employed, are expensive. Thus, it is necessary to make an evidence-based medicine approach in this particular kind of illness to obtain the best benefit/cost ratio . In this current review, we analyzed the different drugs and thera peutic schedules, which are used in the chronic viral hepatitis $B$ and $C$, and as well as their obtained response.
\end{abstract}

KEY WORDS: Randomized controlled trials. Chronic hepatitis $C$. Chronic hepatitis B. Treatment.

Ledro Cano D, Gómez Parra M, Jiménez Sáenz M, Ledro Molina D, Herrerías Gutiérrez JM. Tratamiento de las hepatitis virales crónicas B y C según la medicina basada en la evidencia. An Med Interna (Madrid) 2000; 17: 660-664.

\section{INTRODUCCIÓN}

En todo el mundo, la hepatitis aguda viral es la primera causa de enfermedad hepática aguda y de ictericia. La hepatitis crónica viral es la principal causa de cirrosis hepática y de hepatocarcinoma.

Importantes avances se han producido en el campo de la prevención mediante vacunaciones y medidas de salud publica, sin embargo no existen tratamientos, totalmente satisfactorios para ningún tipo de hepatitis (1).

\section{HEPATITIS CRÓNICA POR VIRUS B}

La terapia de la hepatitis crónica por virus B es actualmente no satisfactoria. El único agente que ha demostrado tener un efecto beneficioso duradero es el alfa-interferón (2). La terapia con interferón conduce a una rápida disminución de los niveles séricos de DNA viral e induce una remisión duradera de la enfermedad en una proporción de los pacientes. Múltiples ensayos randomizados y controlados han demostrado que un régimen de 4 a 6 meses de alfa-interferón induce una pérdida del AgHBe y del DNA viral del suero y una remisión de larga duración entre el 25 y el $40 \%$ de los pacientes (37). Las indicaciones del tratamiento con interferón de la hepatitis crónica B son la elevación persistente de los niveles de las transaminasas, la presencia del AgHBs; AgHBe y del DNA viral en suero, hepatitis crónica, confirmada por histología y la enfermedad hepática compensada. El régimen recomendado es de 5 millones de unidades diarias o 10 millones de unidades, tres veces por semana, subcutánea. Este régimen permite una remisión a largo plazo en el $40 \%$ de los pacientes

Trabajo aceptado: 20 de Diciembre de 1999

Correspondencia: Diego Ledro Cano. Virgen de Luján $454^{\circ} \mathrm{C}$ - 41011 Sevilla 
(8). Los pacientes con el mutante AgHBe negativo necesitan regímenes más largos en el tiempo porque de lo contrario, recidivan (9-11). El tratamiento con interleuquina-2 no ha sido efectivo en el tratamiento de la hepatitis crónica B (12). Análisis multivariados retrospectivos han demostrado la existencia de factores predictivos de buena respuesta al tratamiento de la hepatitis crónica B mediante interferón, tales como un alto nivel de transaminasas séricas (13), corta evolución de la enfermedad, enfermedad activa con fibrosis, niveles bajos de DNA viral, ausencia de inmunosupresión $(8,14)$. El tratamiento con alfainterferón ocasiona múltiples efectos secundarios, la mayoría autolimitados y leves. Entre los graves se encuentran depresión, psicosis, septicemia, insuficiencia cardiaca congestiva, insuficiencia renal aguda y exacerbación de enfermedades autoinmunes (15). Las formas atípicas de la enfermedad, la enfermedad hepática avanzada, los enfermos transplantados y los enfermos con otras patologías importantes no son candidatos a la terapia con interferón, por lo que nuevas opciones terapéuticas son necesarias $(16,17)$. En un estudio realizado con el inmunodulador biológico Timosina alfa 1 (Talfa), los resultados fueron alentadores (18). Existe una vacuna eficaz en el tratamiento de la infección crónica por el virus B de la hepatitis (19). En los pacientes pediatricos, también se ha demostrado el efecto beneficioso del interferón alfa $(20,21)$. Un estudio randomizado y controlado en pacientes con hepatitis crónica B con antígeno HB e positivo, utilizando diversas dosis de lamivudina, mostró resultados beneficiosos (22). La lamivudina parece especialmente indicada en pacientes sometidos a inmunosupresión por transplante de órganos sólidos (23). Recientemente han surgido resistencias a la lamivudina, achacables a mutaciones genéticas (24).

\section{HEPATITIS CRÓNICA POR VIRUS C}

El único agente de beneficio probado en esta enfermedad es el interferón alfa. Antes de descubrirse el virus C, ya se conocía el efecto beneficioso del interferón (25), que fue constatado con los estudios serológicos (26). Por otra parte la erradicación del virus a largo plazo, provocaba una respuesta sostenida (27). Por todo ello varios estudios aleatorizados y controlados de varios regímenes y formas de terapia con interferón alfa de esta enfermedad han sido realizados (28-30). La mayoría de estos estudios mostraron que un régimen prolongado de interferón provoca un descenso en los niveles séricos de transaminasas en la mayoría de los pacientes y normalización de los valores en el 40 a $50 \%$ de los casos. Sin embargo al finalizar los tratamientos, una alta proporción de los respondedores iniciales recidibavan con recuperación de los niveles de transaminasas del inicio del tratamiento. Dosis más altas de interferón alfa solo conducen a ligeros aumentos en la tasa de respuesta. Sin embargo regímenes más largos de 12 y 18 meses, han sido asociados normalmente con un mayor porcentaje de respuesta sostenida $(31,32)$. Una revisión de los trabajos publicados sobre el tratamiento de la hepatitis crónica $\mathrm{C}$ con interferón alfa, permitió a un grupo de expertos reunidos bajo una conferencia de desarrollo de consenso del NIH, afirmar un pauta de tratamiento con interferón alfa de tres millones de unidades tres veces por semana durante un año (33). El grupo de expertos del consenso recomendó el uso de interferón en los pacientes con valores de transaminasas elevados en suero, persistencia del ARN viral en suero y presencia de fibrosis, o al menos grados moderados de inflamación y necrosis en la biopsia hepática (34). En el caso de los pacientes pediatricos, ancianos y con hepatitis leve, la decisión de tratar se basará en el balance entre los efectos adversos y beneficiosos, evaluando cada caso en particular. Sin embargo, son numerosos estudios de tratamiento de esta enfermedad con interferón en la edad pediatrica $(35,36)$. No se recomienda el uso en pacientes con hepatitis crónica $\mathrm{C}$ con valores de transaminasas normales (37). El tratamiento de interferón ha de ser instaurado con precaución en los pacientes neurológicos o psiquiátricos. Existe un grupo de pacientes tratados con interferón alfa, en los cuales existe una normalización de los valores de transaminasas aunque las viremias son persistentemente positivas (38). Biopsias de estos pacientes muestran mejoría de la inflamación y de la necrosis, aunque finalmente recidivaban (39). Estos hallazgos apoyan el tratamiento a largo plazo con interferón alfa en los pacientes sin aclaramiento viral del suero, por la propiedad de este fármaco de disminuir la actividad de la enfermedad. La monitorización de la respuesta al tratamiento debe realizarse con los niveles séricos de transaminasas y la detección en suero del RNA viral. Existen controversias sobre el momento en el cual, se deben valorar ambos parámetros, si bien entre cuatro y seis meses parece razonable, con el objetivo de evaluar la respuesta al tratamiento y decidir su continuación hasta el final del periodo de tratamiento o la suspensión temprana $(33,40)$. Varios tipos de interferónes comerciales están disponibles, aunque todos con similar eficacia, si bien estudios desarrollados para comparar estos preparados, muestran ligeras ventajas de unos sobre otros (41-46). Los efectos indeseables del tratamiento de la hepatitis crónica $\mathrm{C}$ con interferón alfa son similares a los efectos aparecidos durante el tratamiento de la hepatitis crónica B (15), aunque de menor severidad e intensidad, dadas las menores dosis y duración del tratamiento. De especial importancia, son las exacerbaciones de la hepatitis que exigen una retirada del tratamiento, que son debidas al empeoramiento de la enfermedad hepática autoinmune (47). Los tratamientos prolongados ocasionan síntomas psiquiátricos. Estudios sobre el tratamiento de la hepatitis crónica $\mathrm{C}$ con inteferon alfa durante 6 meses, muestran una tasa global de respuesta sostenida del 8 al 12\% (42). Tratamientos más prolongados mejoran la tasa hasta respuestas desde el 16 al 24\% (48-50). Incrementos en la dosis, mejoran escasamente estos resultados $(28,51,52)$. Los factores que han sido asociados con una mayor respuesta son edad, sexo, duración de la enfermedad, fuente de la infección, metabolismo del hierro, fibrosis hepática, cirrosis hepática, genotipos virales, viremia y diversidad de cuasiespecies $(53,54)$. Diversos y amplios estudios, utilizando análisis multivariantes, han demostrado que existen cinco factores predictivos de buena respuesta: edad joven, ausencia de cirrosis o fibrosis mínima, bajos niveles de hierro intrahepáticos previos al tratamiento, viremia baja, genotipos virales 2 y 3 . Ningún factor aislado o combinación de los mismos, es totalmente predictivo de respuesta. Son útiles a la hora de aconsejar al paciente sobre la necesidad de tratamiento y las posibilidades de respuesta. Ponen de manifiesto la necesidad de estratificar a los pacientes en los ensayos clínicos. La presencia de inmunosupresion por transplante o por SIDA, insuficiencia renal, las complicaciones extrahepáticas de la infección del virus $\mathrm{C}$, niveles normales de transaminasas son factores predictivos negativos de respuesta (55). En el momento actual, el tratamiento en estos pacientes debe ser considerado como experimental y debe llevarse a cabo, dentro de ensayos clínicos alea- 
torizados y controlados. El porcentaje relativamente bajo de respuesta sostenida a la terapia con interferón alfa de la hepatitis crónica $\mathrm{C}$ ha conducido a la investigación de otros agentes de uso potencial en esta enfermedad. Este avance ha sido entorpecido por la ausencia de sistemas in vivo e in vitro para evaluar agentes contra esta enfermedad (56). Un estudio analiza el uso de extracto tímico por vía oral, en pacientes que han recibido tratamiento con interferón alfa, sin poder demostrar ningún tipo de beneficio (57). Sin embargo, el uso de fostatidilcolina poliinsaturada acompañando al tratamiento con interferón y al terminar este, es beneficioso en la prevención de la recidiva de la enfermedad (58). Un fármaco muy usado en Japón es la glicirrizina con resultados alentadores (59). Varios estudios han utilizado el ácido ursodesoxicólico asociado a interferón (60-63) o sólo (64), señalando el papel adyuvante de este fármaco en la mejora del cuadro bioquímico de esta enfermedad. El uso de factores estimulantes de colonias sólos o asociados al interferón alfa no producen ningún efecto beneficioso (65). La deplección del hierro hepático mejora el índice de respuesta sostenida al interferón (66). El uso de alfa1 timosina añadida al interferón, produce una mejoría bioquímica, histologica y vírica importante, aunque se halla en fase experimental (67) y algún estudio señala resultados no beneficiosos (68). En el momento actual, el único agente antiviral con efectos prometedores en el tratamiento de la hepatitis crónica $\mathrm{C}$ es la ribavirina, que es un fármaco antiviral de amplio espectro de administración oral, que ha sido utilizado con éxito en la infección por el virus sincitial respiratorio en pacientes pediatricos. El tratamiento con ribavirina durante doce meses o más, va seguido de una mejoría histologica y disminución gradual de los niveles de transaminasas en suero (69). Desgraciadamente, al finalizar el tratamiemto, existe una recidiva en la mayoría de los pacientes con regreso a los niveles de transaminasas previos al inicio del tratamiento y la viremia es persistente. Estos hechos apuntan a la posibilidad de un efecto supresivo durante el tratamiento, pero de escasa duración, una vez finalizado el mismo. El tratamiento largo y mantenido con ribavirina debe ser evaluado, sobre todo en lo que se refiere a la seguridad y eficacia, valorando efectos beneficiosos y adversos, con suma cautela. Sin embargo, más prometedores resultados se han obtenido con la combinación de interferón alfa con ribavirina, incluso en pacientes transplantados. Alcanzando altas tasas de respuesta sostenida. Incluso en algunos pacientes, a pesar de viremia persistentemente positiva, se han mantenido valores de transaminasas normales, aun con monoterapia con ribavirina (70-76). El único efecto secundario de la ribavirina es la aparición de anemia hemolítica, de la cual, la mayoría de los pacientes se recuperan tras tres a seis semanas de tratamiento, si bien en algunos puede ser grave y sintomática. Por ello en pacientes con enfermedad arteriosclerotica, insuficiencia renal o anemia previas, su uso debe evitarse, así como en las embarazadas, por ser teratogenica.

\section{Bibliografía}

1. Hoofnagle JH, Di Bisceglie AM. Treatment of chronic viral hepatitis. $\mathrm{N}$ Eng J Med 1997; 336: 347-356.

2. Alter MJ, Mast EE. The epidemiology of viral hepatitis in the United States. Gastroenterol Clin N Am 1994; 23:437-455.

3. Alexander GJM, Brahm J, Fagan EA, Smith HM, Daniels HM, Eddleston ALWF, Williams R. Loss of HbsAg with interferon therapy in chronic hepatitis B virus infection. Lancet 1987; i: 66-68

4. Hoofnagle JH, Peters MG, Mullen KD, Jones DB, Rustgi V, Di Bisceglie A, Hallahan C, Park Y, Meschievitz C, Jones EA. Randomized controlled trial of recombinant human alpha-interferon in patients with chronic hepatitis B. Gastroenterology 1988; 95: 1318-1325

5. Perrillo RP, Schiff E, Davis GL, Bodenheimer HC Jr, Lindsay K, Payne J, Dienstag JL, O'Brien C, Tamburro C, Jacobson IM, Sampliner R, Feit D, Lefkowitch J, Kuhns M, Meschievitz C, Sanghvi B, Albrecht J, Gibas A and Hepatitis Interventional Therapy Group. A randomized controlled trial of interferon alpha- $2 b$ alone and after prednisone withdrawal for the treatment of chronic hepatitis B. N Eng J Med 1990; 323 : 295-301.

6. Lok ASF, Wu P-C, Lai C-L, Lau JYN, Leung EKY, Wong LSK, Ma OCK, Lauder IJ, Ng CPL, Chung H-T. A controlled trial of interferon with or without prednisone priming for chronic hepatitis B. Gastroenterology 1992; 102: 2091-2097.

7. Krogsgaard K. The long-term effect of treatment with interferon-alpha $2 \mathrm{a}$ in chronic hepatitis $\mathrm{B}$. The Long-Term Follow-up Investigator Group. The European Study Group on Viral Hepatitis (EUROHEP). Executive Team on Anti-Viral Treatment. J Viral Hepat 1998; 5(6): 389- 97.

8. Wong DKH, Cheung AM, O'Rourke K, Naylor CD, Detsky AS, Heathcote J Effects of alpha-interferon treatment in patients with hepatitis B e antigen - positive chronic hepatitis B: a meta-analysis. Ann Intern Med 1993; 119: 312-323.

9. Brunetto MR, Giarin M, Saracco G, Oliveri F, Calvo P, Capra G, Randone A, Abate ML, Manzini P, Capalbo M, Piantino P, Verme G, Boni- no F. Hepatitis B virus unable to secrete e antigen and response to interferon in chronic hepatitis B. Gastroenterology 1993; 105: 845-850.

10. Hadziyannis SJ. Hepatitis B e antigen negative chronic hepatitis B: From clinical recognition to pathogenesis and treatment .Viral Hepatitis Rev 1995; 1: 7-15.

11. Lampertico P, Del Ninno E, Manzin A, Donato MF, Rumi MG, Lunghi G, Morabito A, Clementi M, Colombo M. A randomized, controlled trial of a 24-month course of interferon alfa $2 \mathrm{~b}$ in patients with chronic hepatitis B who had hepatitis B virus DNA without hepatitis B e antigen inserum. Hepatology 1997; 26 (6): 1621-5.

12. Artillo S, Pastore G, Alberti A, Milella M, Santantonio T, Fattovich G, Giustina G, Ryff JC, Chaneac M, Bartolome J, Carreno V. Doubleblind, randomized controlled trial of interleukin-2 treatment of chronic hepatitis B. J Med Virol 1998; 54 (3): 167-72.

13. Lok AS, Ghany MG, Watson G, Ayola B. Predictive value of aminotransferase and hepatitis B virus DNA levels on response to interferon therapy for chronic hepatitis B. J Viral Hepat 1998; 5 (3): 171-8.

14. Lau D T-Y, Comanor L, Minor JM, Everhart JE, Wuestehube LJ, Hoofnagle JH Statistical models for predicting a beneficial response to interferon-alpha in patients with chronic hepatitis B. J Viral Hepatitis 1998; 5: 105-114.

15. Dushieko G. Side effects of alpha-interferon in chronic hepatitis C. Hepatology 1997; 26: 112S-121S.

16. Wright HI, Gavaler JS, Van Thiel DH. Preliminary experience with alp ha-2b interferon therapy of viral hepatitis in liver allograft recipients. Transplantion 1992; 53: 121-124.

17. Marcellin P, Boyer N, Colin JF, Martinot-Peignoux M, Lefort V, Matherson S, Erlinger S, Benhamou J-P. Recombinant alpha-interferon for chronic hepatitis $\mathrm{B}$ in anti-HIV positive patients receiving zidovudine. Gut 1993; 34 (suppl 2): S106.

18. Chien RN, Liaw YF, Chen TC, Yeh CT, Sheen IS. Efficacy of thymosin alpha1 in patients with chronic hepatitis B: a randomized, controlled trial.Hepatology May 27 (5): 1383-7. 
19. Pol S, Couillin I, Michel ML, Driss F, Nalpas B, Carnot F, Berthelot P, Brechot C. Immunotherapy of chronic hepatitis B by anti HBV vaccine. Acta Gastroenterol Belg 1998; 61 (2): 228-33.

20 Vajro P, Migliaro F, Fontanella A, Orso G. Interferon: a meta-analysis of published studies in pediatric chronic hepatitis B. Acta Gastroenterol Belg 1998; 61 (2): 219-23.

21. Sokal EM, Conjeevaram HS, Roberts EA, Alvarez F, Bern EM, Goyens P, Rosenthal P, Lachaux A, Shelton M, Sarles J, Hoofnagle J. Interferon alfa therapy for chronic hepatitis B in children: a multinational randomized controlled trial.Gastroenterology 1998; 114 (5): 988-95.

22. Lai CL, Liaw TY, Leung NWY, Chang TT, Guan R, Tai DI, Ng KY, Wu PC, Dent JC, Gray DF .12 months therapy improves liver histology: Results of a placebo controlled multicenterstudy in Asia. (abstract). J Hepatology 1997; 26: 79

23. Bartholomew MM, Jansen RW, Jeffers LJ, Reddy KR, Johnson LC, Bunzendahl H, Condreay LD, Tzakis AG, Schiff ER, Brown NA. Hepatitis B virus resistance to lamivudine given for recurrent infection after orhtotopic liver transplantion. Lancet 1997; 349: 20-22.

24. Ling R, Mutimer D, Ahmed M, Boxall EH, Elias E, Dusheiko GM, Harrison TJ. Selection of mutations in the hepatitis B virus polymerase during therapy of transplant recipients with lamivudine Hepatology 1996; $24: 711-713$

25. Hoofnagle JH, Mullen KD, Jones DB, Rustgi V, Di Bisceglie A, Peters M, Waggoner JG, Park Y, Jones EA. Treatment of chronic non-A, nonB with recombinant human alpha interferon. N Eng J Med 1986; 315 : $1575-1578$

26. Shindo M, Di Bisceglie AM, Cheung L, Shih JW, Cristiano K, Feinstone SM, Hoofnagle JH. Decrease in serum Hepatitis C viral RNA during alpha-interferon therapy for chronic hepatitis C. Ann Intern Med 1991; 115: 700-704.

27. Shindo M, Di Bisceglie AM, Hoofnagle JH. Long-term follow-up of patients with chronic hepatitis $\mathrm{C}$ treated with alpha-interferon. Hepatology 1992; 15: 1013-1016.

28. Yoshioka K, Yano M, Kusakabe A, Hirofuji H, Fuji A, Kuriki J, Arao M, Murase K, Kidokoro R, Kakumu S. Randomized controlled trial of lymphoblastoid interferon alpha for chronic hepatitis $\mathrm{C}$ (comparison of 9-MU and 6-MU doses). IFN Treatment Group of Affiliated Hospitals of the Third Department of Internal Medicine at Nagoya University School of Medicine. Am J Gastroenterol 1999; 94 (1): 164-8.

29. Ascione A, De Luca M, Canestrini C, Di Costanzo GG, Raimondo G, Longo G, Manns MP, Tillmann HL, Forte GB, Rocco P, Biceglia O, Faleo D, Vinelli F, Cela EM, Amitrano L, Addario L, Gigliotti T. Efficacy of high dose of recombinant alpha $2 b$ interferon on longterm response in chronic hepatitis $\mathrm{C}$ and cirrhosis: prospective randomized multicentre study. Ital J Gastroenterol Hepatol 1998; 30 (5): 517-23.

30. Farrell GC, Bacon BR, Goldin RD. Farrell GC, Bacon BR, Goldin RD. Lymphoblastoid interferonalfa-n1 improves the long-term response to a 6-month course of treatment in chronic hepatitis $\mathrm{C}$ compared with recombinant interferon alfa-2b: results of an international randomized controlled trial. Clinical Advisory Group for the Hepatitis C Comparative Study. Hepatology 1998; 27 (4): 1121-7.

31. Poynard T, Leroy V, Cohard M, Thevenot T, Mathurin P, Opolon P, Zarski JP. Meta-analysis of interferon randomized trials in the treatment of viral hepatitis C: effects of dose and duration. Hepatology 1996; 24(4): 778-89.

32. RL Jr, Emerson SS. Therapy of hepatitis C: meta-analysis of interferon alfa-2b trials. Hepatology 1997; 26 (3 Suppl 1): 83S-88S.

33. National Institutes of Health consensus development conference panel: Management of hepatitis C. Hepatology 1997; 26: 2S-10S.

34. Heintges T, Mohr L, Hensel F, Petry W, Borchard F, Haussinger D, Niederau $C$ Value of liver biopsy prior to interferon therapy for chronic viral hepatitis. Dig Dis Sci 1998; 43 (7): 1562-5.

35. Bortolotti F, Giacchino R, Vajro P, Barbera C, Crivellaro C, Alberti A, Nebbia G, Zancan L, De Moliner L, Bertolini A, et al Recombinant interferon-alfa therapy in children with chronic hepatitis C. Hepatology 1995; 22 (6): 1623-7.

36. Vegnente A, Iorio R, Pensati P Treatment of pediatric hepatitis C: results and perspectives.Acta Gastroenterol Belg 1998; 61 (2): 235-6.

37. Sangiovanni A, Morales R, Spinzi G, Rumi M, Casiraghi A, Ceriani R, Colombo E, Fossati M, Prada A, Tavani E, Minoli G Interferon alfa treatment of HCV RNA carriers with persistently normal transaminase levels: a pilot randomized controlled study. Hepatology 1998; 27 (3): 853-6.

38. Lau JYN, Mizokami M, Ohmo T, Diamond DA, Kniffen J, Davis GL. Discrepancy between biochemical and virological responses to interferon alpha in chronic hepatitis C. Lancet 1993; 342: 1208-1209.
39. Camma C, Di Marco V, Lo Iacono O, Almasio P, Giunta M, Fuschi P, Vaccaro A, Fabiano C, Magrin S, DI Stefano R, Bomura C, Pagliaro L, Craxi A. Long-term course of interferon-treated chronic hepatitis C. J Hepatol 1998; 28: 531-537.

40. Keeffe E, Smith C, Lyche K, CIFN study group. Predicitive values of timing of ALT and HCV RNA responses in defining patients who will have a virologic-sustained response after six months of interferon therapy (abstract). Gastroenterology 1998; 114: A1271.

41. Calleri G, Colombatto P, Gozzelino M, Chieppa F, Romano P, Delmastro B, Macor A, Cariti G, Brunetto MR, Grillone W, Bonino F. Natural beta interferon in acute type-C hepatitis patients: a randomized controlled trial. Ital J Gastroenterol Hepatol 1998; 30 (2): 181-4.

42. Farrell GC, Bacon BR, Goldin RD Lymphoblastoid interferon alfa-n1 improves the long-term response to a 6-month course of treatment in chronic hepatitis $\mathrm{C}$ compared with recombinant interferon alfa- $2 \mathrm{~b}$ : results of an international randomized controlled trial. Clinical Advisory Group for the Hepatitis C Comparative Study. Hepatology 1998; 27 (4): 1121-7.

43. Keeffe EB, Hollinger FB. Keeffe EB, Hollinger FB. Therapy of hepatitis C: consensus interferon trials. Consensus Interferon Study Group. Hepatology 1997; 26 (3 Suppl 1): 101S-107S.

44. Gaeta GB, Di Virgilio D, Russo G, Stornaiuolo G, Nicolella U, Colella F, Grimaldi M, Pasquale G, Giusti G Human leucocyte interferon-alpha in chronic hepatitis $\mathrm{C}$ resistant to recombinant or lymphoblastoid interferon-alpha: a randomized controlled trial. J Viral Hepat 1997; 4 (3): 209-14.

45. Castro A, Suárez D, Inglada L, Carballo E, Dominguez A, Diago M, Such J, Del Olmo JA, Pérez-Mota A, Pedreira J, Quiroga JA, Carreno V Multicenter randomized, controlled study of intramuscular administration of interferon-beta for the treatment of chronic hepatitis C. J Interferon Cytokine Res 1997; 17 (1): 27-30.

46. Simon DM, Gordon SC, Kaplan MM, Koff RS, Regenstein F, Everson G, Lee YM, Weiner F, Silverman A, Plasse T, Fedorczyk D, Liao MJ Treatment of chronic hepatitis $\mathrm{C}$ with interferon alfa-n3: a multicenter, randomized, open-label trial.Hepatology 1997; 25 (2): 445-8.

47. Shindo M. Di Bisceglie AM, Hoofnagle JH. Acute exacerbation of liver disease during interferon alfa therapy for chronic hepatitis C. Gastroenterology 1992; 102: 1406-1408.

48. Carithers RL Jr, Emerson SS Therapy of hepatitis C: meta-analysis of interferon alfa-2b trials. Hepatology 1997; 26 (3 Suppl 1): 83S-88S.

49. Poynard T, Leroy V, Cohard M, Thevenot T, Mathurin P, Opolon P, Zarski JP Meta-analysis of interferon randomized trials in the treatment of viral hepatitis C: effects of dose and duration Hepatology 1996; 24 (4): 778-89.

50. Kasahara A, Hayashi N, Hiramatsu N, Oshita M, Hagiwara H, Katayama K, Kato M, Masuzawa M, Yoshihara H, Kishida Y, et al. Ability of prolonged interferon treatment to suppress relapse after cessation of therapy in patients with chronic hepatitis $\mathrm{C}$ : a multicenter randomized controlled trial. Hepatology 1995; 21 (2): 291-7.

51. Di Marco V, Lo Iacono O, Camma C, Almasio PL, Vaccaro A, Fuschi P, Giunta M, Fabiano C, Pagliaro L, Craxi A A randomized controlled trial of high-dose maintenance interferon therapy in chronic hepatitis $\mathrm{C}$. J Med Virol 1997; 51 (1): 17-24.

52. Ascione A, De Luca M, Canestrini C, Di Costanzo GG, Raimondo G, Longo G, Manns MP, Tillmann HL, Forte GB, Rocco P, Biceglia O, Faleo D, Vinelli F, Cela EM, Amitrano L, Addario L, Gigliotti T. Efficacy of high dose of recombinant alpha $2 \mathrm{~b}$ interferon on long term response in chronic hepatitis $\mathrm{C}$ and cirrhosis: prospective randomized multicentre study. Ital J Gastroenterol Hepatol 1998; 30(5): 517-23

53. Saracco G, Rizzetto M. Predictors of response to interferon therapy. Dig Dis Sci 1996; 41 (12 Suppl): 115S-120S.

54. Davis GL, Lau JYN. Factors predictive of a beneficial response to therapy in hepatitis. Hepatology 1997; 26: 112S-127S.

55. Féray C, Samuel D, Gigou M, Paradis V, David MF, Lemonnier C, Reynès, Bismuth H. An open trial of interferon alfa recombinant for hepatitis $\mathrm{C}$ after liver transplantation: antiviral effects and risk of rejection. Hepatology 1995; 22: 1084-1089.

56. Shimizu YK, Iwamoto A, Hijikata M, Purcell RH, Yoshikura H. Evidence for in vitro replication of hepatitis $\mathrm{C}$ virus genome in a human $\mathrm{T}$ cell line. Proc Natl Acad Sci USA 1992; 89: 5477-5481.

57. Raymond RS, Fallon MB, Abrams GA Oral thymic extract for chronic hepatitis $\mathrm{C}$ in patients previously treated with interferon. A randomized, double-blind, placebo-controlled trial. Ann Intern Med 1998; 129 (10): 797-800. 
58. Niederau C, Strohmeyer G, Heintges T, Peter K, Gopfert E Polyunsatu rated phosphatidyl-choline and interferon alpha for treatment of chronic hepatitis B and C: a multi-center, randomized, double blind, placebocontrolled trial. Leich Study Group. Hepatogastroenterology 1998; 45 (21): 797-804

59. van Rossum TG, Vulto AG, de Man RA, Brouwer JT, Schalm SW Review article: glycyrrhizin as a potential treatment for chronic hepatitis C.Aliment Pharmacol Ther 1998; 12(3): 199-205.

60. Abdelmalek MF, Harrison ME, Gross JB Jr, Poterucha JJ, Gossard AA, Spivey JR, Rakela J, Lindor KD Treatment of chronic hepatitis C with interferon with or without ursodeoxycholic acid: a randomized prospective trial.J Clin Gastroenterol 1998; 26 (2): 130-4.

61. Kiso S, Kawata S, Tamura S, Imai Y, Inui Y, Nagase T, Maeda Y, Yamasaki E, Tsushima H, Igura T, Himeno S, Seki K, Matsuzawa Y Efficacy of combination therapy of interferon-alpha with ursodeoxycholic acid in chronic hepatitis C: a randomized controlled clinical trial. J Gastroenterol 1997; 32 (1): 56-62.

62. Angelico M, Gandin C, Pescarmona E, Rapicetta M, Del Vecchio C, Bini A, Spada E, Baroni CD, Capocaccia L Recombinant interferonalpha and ursodeoxycholic acid versus interferon- alpha alone in the treatment of chronic hepatitis $\mathrm{C}$ : a randomized clinical trial with long-term follow-up. Am J Gastroenterol 1995; 90 (2): 263-9.

63. Boucher E, Jouanolle H, Andre P, Ruffault A, Guyader D, Moirand R, Turlin B, Jacquelinet C, Brissot P, Deugnier Y Interferon and ursodeoxycholic acid combined therapy in the treatment of chronic viral $\mathrm{C}$ hepatitis: results from a controlled randomized trial in 80 patients Hepatology 1995; 21 (2): 322-7.

64. Takano S, Ito Y, Yokosuka O, Ohto M, Uchiumi K, Hirota K, Omata M A multicenter randomized controlled dose study of ursodeoxycholic acid for chronic hepatitis C. Hepatology 1994; 20 (3): 558-64.

65. Shiffman ML, Hofmann CM, Luketic VA, Sanyal AJ Use of granuloc te macrophage colony stimulating factor alone or in combination with interferonalpha-2b for treatment of chronic hepatitis C.J Hepatol 1998; 28 (3): 382-9.

66. Fong TL, Han SH, Tsai NC, Morgan TR, Mizokami M, Qian D, Phan C, Goad K, Redeker AG A pilot randomized, controlled trial of the effect of iron depletion on long-term response to alpha-interferon in patients with chronic hepatitis C. J Hepatol 1998; 28 (3): 369-74.

67. Sherman KE, Sjogren M, Creager RL, Damiano MA, Freeman S, Lewey S, Davis D, Root S, Weber FL, Ishak KG, Goodman ZD. Combination therapy with thymosin alphal and interferon for the treatment of chronic hepatitis $\mathrm{C}$ infection: a randomized, placebo-controlled double-blind trial. Hepatology 1998; 27(4): 1128-35.

68. Andreone P, Cursaro C, Gramenzi A, Buzzi A, Covarelli MG, Di Giammarino L, Miniero R, Arienti V, Bernardi M, Gasbarrini G A doubleblind, placebo-controlled, pilot trial of thymosin alpha 1 for the treatment of chronic hepatitis C Liver 1996; 16 (3): 207-10.

69. Di Bisceglie AM, Conjeevaram HS, Fried MW, Sallie R, Park Y, Yurdaydin C, Swain M, Kleiner DE, Mahaney K, Hoofnagle JH Ribavirin as therapy for chronic hepatitis C. A randomized, double-blind, placebo-controlled trial. Ann Intern Med 1995 15; 123 (12): 897-903.

70. Poynard T, Moussalli J, Ratziu V, Thevenot T, Regimbeau C, Opolon P, Horsmans Y, Brenard R, Closon M, Fevery J, Hautekeete M Is antivira treatment (IFN alpha and/or ribavirin) justified in cirrhosis related to hepatitis C virus? Societe Royale Belge de Gastroenterologie. Acta Gastroenterol Belg 1998; 61 (4): 431-7.

71. Barbaro G, Di Lorenzo G, Soldini M, Giancaspro G, Bellomo G, Belloni G, Grisorio B, Annese M, Bacca D, Francavilla R, Rizzo G, Barbarini $G$ Interferon-alpha-2B and ribavirin in combination for chronic hepatitis $\mathrm{C}$ patients not responding to interferon-alpha alone: an Italian multicenter, randomized, controlled, clinical study. Am J Gastroenterol 1998; 93 (12): 2445-51.

72. Schalm SW Adding ribavirin to interferon alpha- $2 b$ for chronic hepatitis $C$ infection increased virological response and nausea.Gut 1998; 43 (5): 602.

73. Wedemeyer H, Jackel E, Wedemeyer J, Frank H, Schuler A, Trautwein $\mathrm{C}$, Manns MP Is combination therapy of chronic hepatitis $\mathrm{C}$ with interferon alpha and ribavirin in primary interferon nonresponders indicated? An analysis of personal experiences and review of the literature. Z Gastroenterol 1998; 36 (9): 819-27.

74. Sostegni R, Ghisetti V, Pittaluga F, Marchiaro G, Rocca G, Borghesio E, Rizzetto M, Saracco G Sequential versus concomitant administration of ribavirin and interferon alfa-n 3 in patients with chronic hepatitis $\mathrm{C}$ not responding to interferon alone: results of a randomized, controlled trial. Hepatology 1998; 28 (2): 341-6.

75. Reichard O, Schvarcz R, Weiland O Therapy of hepatitis C: alpha interferon and ribavirin. Hepatology 1997; 26 (3 Suppl 1): 108S-111S

76. Schalm SW, Hansen BE, Chemello L, Bellobuono A, Brouwer JT, Weiland O, Cavalletto L, Schvarcz R, Ideo G, Alberti A Ribavirin enhances the efficacy but not the adverse effects of interferon in chronic hepatitis C. Meta-analysis of individual patient data from European centers. J Hepatol 1997; 26 (5): 961-6. 